\title{
Hemangioma de úvula: relato de um caso
}

\section{Hemangioma of uvula: onecasereport}

Palavras-chave: hemangioma, úvula, bisturi-radiofreqüência.

Key words: hemangioma, uvula, radio frequency cautery.

\section{Resumo / Summary}

hemangioma é o mais comum tumor de origem vascular, benigno, freqüentemente diagnosticado em crianças, e com predileção pela cabeça e pescoço, mas que raramente tem origem na úvula. Neste órgão, apresenta risco de traumatismo local e sangramento. Este artigo relato um caso de hemangioma de úvula num adulto jovem com disfagia orofaríngea progressiva e sensação de corpo estranho na garganta, tratado com sucesso por ressecção cirúrgica do tumor com bisturi de radiofreqüência.
The

he hemangioma is the most common tumor of the vascular origin, benign, frequently observed in children, and with preference for head and neck, but rarely has uvular origin. In this organ, it presents potential risk of local trauma and hemorrhage. The present article reports a case of hemangioma of uvula in adult with progressive oropharyngeal dysphagia and sensation of foreign body in the throat, treated with success by surgical resection of the tumor using radio frequency cautery.

${ }^{1}$ Médico Colaborador do Serviço de O torrinolaringologia do Hospital das Clínicas - Universidade Federal da Bahia.

${ }_{2}^{2}$ Médico Colaborador do Serviço de Otorrinolaringologia do Hospital das Clínicas - Universidade Federal da Bahia.

${ }_{3}^{3}$ Coordenador da Divisão de Cirurgia de Cabeça e Pescoço do Serviço de O torrinolaringologia do Hospital das Clínicas - Universidade Federal da Bahia.

4 Professor Doutor da Disciplina de Otorrinolaringologia da Faculdade de Medicina da Universidade Federal da Bahia e Chefe do Serviço de O torrinolaringologia do Hospital das Clínicas da Universidade Federal da Bahia.

Trabalho realizado pelo Serviço de O torrinolaringologia do Hospital das Clínicas da Universidade Federal da Bahia. Endereço para Correspondência: Fernando P. G. Sobrinho - Cd. Rec. dos Pássaros, R3, B29-A, Ap.301 Cabula 41150-050 Salvador BA Tel (0xx71) 257-0226 - E-mail: fpgsobrinho@bol.com.br

Artigo recebido em 25 de setembro de 2002. Artigo aceito em 07 de novembro de 2002 


\section{INTRODUÇÃO}

0 hemangioma é o mais comum de todos os tumores de origem vascular, histologicamente benigno, sendo diagnosticado principalmente na infância ${ }^{1}$. No segmento cefálico, tem localização preferencial na face, mucosa bucal, lábios e língua, raramente acometendo a úvula ${ }^{2}$. Sua localização pode torná-lo vulnerável à hemorragia por traumatismo local ${ }^{3}$. Os hemangiomas da cavidade oral e orofaringe também podem apresentar dificuldades peculiares para uma abordagem operatória, quando indicada, sobretudo pela limitada via de acesso cirúrgico e risco de sangramento ${ }^{3}$.

0 objetivo do presente trabalho é relatar um caso de hemangioma obstrutivo de úvula num adulto jovem tratado eficazmente com ressecção cirúrgica em bloco com bisturi de radiofreqüência e revisar características relacionadas aos hemangiomas e malformações vasculares.

\section{RELATO DE CASO}

Paciente do sexo masculino, mulato, estudante, com idade de 21 anos, referindo sensação de corpo estranho na garganta há 2 anos, com piora progressiva, sobretudo nos últimos quatro meses, evoluindo para disfagia orofaríngea. Nega sangramento, dor ou febre. Antecedente médico irrelevante. Critérios para inclusão em grupo de risco para doenças sexualmente transmissíveis ausentes.

o exame da orofaringe revelou uma úvula com grande volume e superfície irregular, apresentando uma lesão de aspecto angiomatoso, violáceo-escuro, comprometendoa em toda sua extensão, com consistência amolecida, indolor à manipulação (Figura 1). A inspeção, a rinoscopia posterior e a palpação cuidadosa do palato mole e do tumor sugeriram o diagnóstico de hemangioma cavernoso limitado à úvula. 0 exame físico segmentar restante, incluindo o cérvico-facial, não mostrou anormalidades. Um estudo por tomografia computadorizada com e sem contraste foi obtido e não modificou as impressões evidenciadas ao exame físico da orofaringe. A sorologia para o HIV foi negativa.

0 paciente foi submetido a procedimento cirúrgico sob anestesia geral com intubação nasotraqueal. Foi realizada ressecção em bloco de toda úvula com bisturi de radiofreqüência, com mínimo sangramento intra-operatório. Evoluiu sem intercorrências no pós-operatório imediato. 0 seguimento ambulatorial revelou um bom resultado cirúrgico (Figura 2). A avaliação histopatológica concluiu tratar-se de hemangioma cavernoso (Figura 3).

\section{Hemangioma e malformação vascular}

0 hemangioma é o mais comum de todos os tumores vasculares ${ }^{1}$. É o tumor mais freqüente na infância, com cerca de $73 \%$ dos casos diagnosticados ainda no primeiro ano de vida ${ }^{4}$. Acredita-se que acomete $1 \%$ das crianças caucasianas ${ }^{5}$, sendo mais freqüente em brancos que em negros ${ }^{1}$, e com

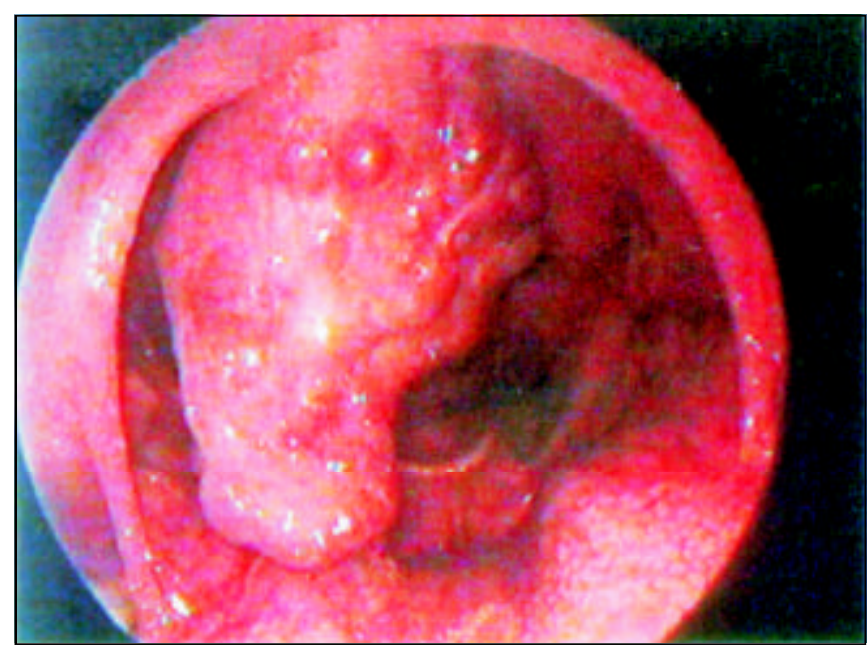

Figura 1. Orofaringoscopia revela comprometimento da úvula em toda extensão por lesão de natureza vascular, com aspecto "bag of worms", sugestiva de hemangioma cavernoso.

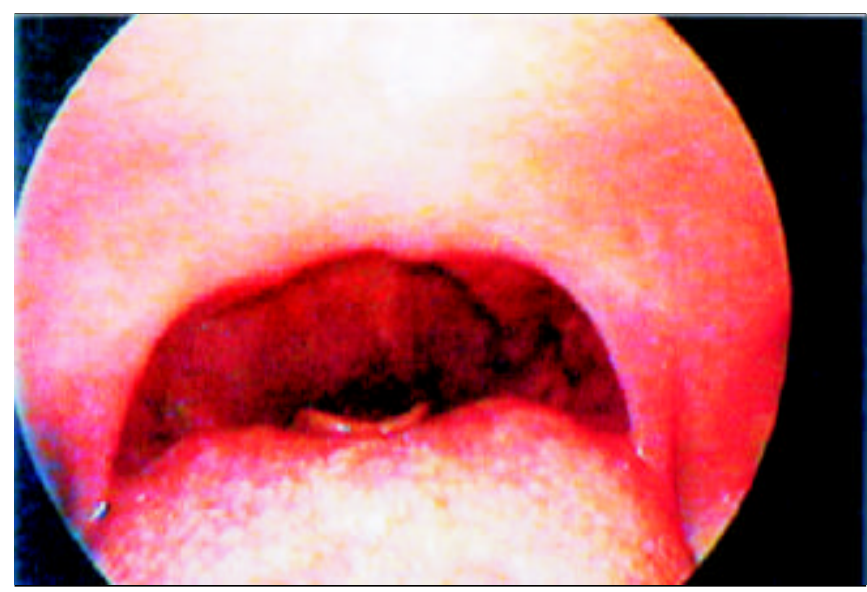

Figura 2. Na orofaringoscopia pós-operatória, observa-se ausência de lesão vascular e completa cicatrização de ferida cirúrgica.

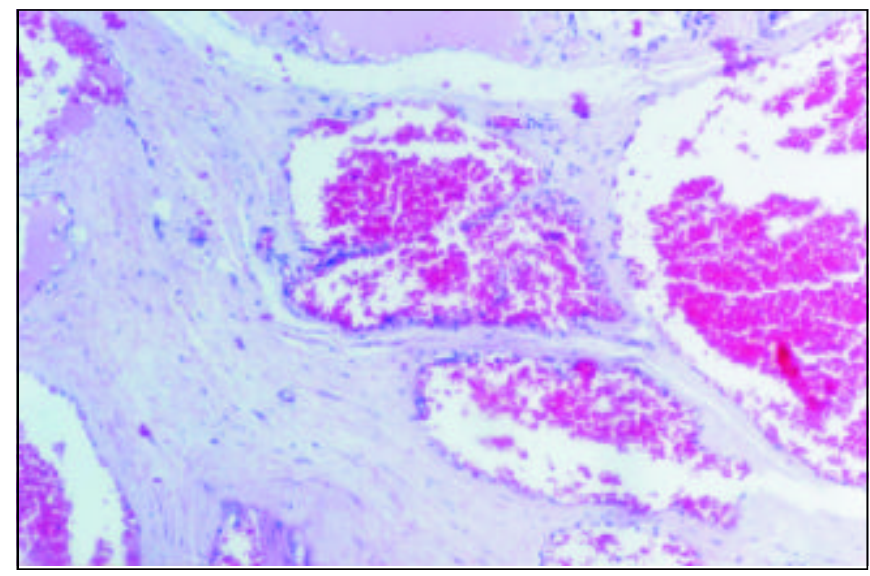

Figura 3. A histopatologia mostra vasos ectásicos, de parede vascular delgada, entremeado por tecido fibroso. 
prevalência 3 vezes maior no sexo feminino. Os hemangiomas são mais comuns na cabeça e pescoço ${ }^{5}$, embora possam acometer qualquer outro segmento do corpo.

A princípio, o hemangioma habitualmente surge como uma circunscrita placa macular ou telangiectasia localizada em pele e/ou mucosas, na infância, sobretudo durante as primeiras seis semanas de vida. Também pode ter origem mais profunda, sub-tegumentar, ou envolver órgãos internos. A lesão pode apresentar um rápido crescimento pós-natal, histologicamente designado de "fase proliferativa"1,4, caracterizada por acentuada hiperplasia endotelial, freqüentemente desenvolvendo-se ao longo de alguns meses, aos quais se segue um lento período de remissão, denominado de "fase involutiva", que geralmente compreende cinco a oito anos ${ }^{1}$ e envolve a deposição progressiva de estroma fibrogorduroso permeando vasos com parede endotelial e lúmen melhor definidos. Este padrão difásico representa um importante parâmetro na diferenciação nosológica entre um hemangioma e uma malformação vascular propriamente dita ${ }^{1}$. Esta última está presente ao nascimento e desenvolve-se conforme o ritmo geral de crescimento do indivíduo, embora possa aumentar anormalmente suas dimensões após traumatismo local, infecção, iatrogenia ou alterações hormonais. A lesão cutaneomucosa tipo "vinho-do-porto" observada na síndrome de Sturge-Osler-Weber é um exemplo de malformação vascular, conforme a classificação biológica das anomalias vasculares proposta por Mulliken e Glowacki ${ }^{6}$.

Embora existam controvérsias, uma outra importante classificação diferencia os hemangiomas em capilares, cavernosos ou mistos $s^{4,5}$. Os p rimeiros seriam caracterizados por pequenos vasos "capilares" com delgada parede vascular, delimitada geralmente por única camada endotelial. Clinicamente podem mostrar-se como lesões superficiais, rasas, róseas a avermelhadas, de consistência firme, freqüentemente com algum grau de lobulação, que associada a uma intensa coloração avermelhada, levou alguns autores a descreverem esta apresentação como hemangioma "em framboesa". Com a involução assumem aspecto enegrecido e posteriormente cinzento e pálido, associados à fibrose tecidual progressiva. Por sua vez, o hemangioma cavernoso é composto por vasos de paredes finas e maior diâmetro, irregulares, com tendência a localizar-se mais profundamente em relação ao tegumento cutaneomucoso e pode acometer órgãos internos e o sistema nervoso. Ao exame físico, tem delimitação menos definida e consistência amolecida à palpação, quando empalidece ao toque. Pode expandir-se com manobras que promovam um maior enchimento venoso, como a de Valsalva. Foi descrita por alguns autores como uma "bolsa de vermes" (bag of worms) $)^{5}$. Finalmente, 0 hemangioma classificado como misto teria características comuns às duas formas descritas.

Atualmente, sobretudo após os trabalhos de Mulliken, para alguns autores o termo hemangioma profundo ou superficial substitui as designações clássicas de cavernoso ou capilar, respectivamente, pois, segundo eles, as características individuais dos hemangiomas descritas pela terminologia clássica poderiam sobrepor-se e ser, na prática clínica, de limitada acurácia nas definições de um caso clínico. Quando a lesão envolvesse concomitantemente camadas superficiais e profundas da pele ou mucosa seria classificada como mista ou capilar-cavernosa.

A grande maioria dos hemangiomas pode ser diagnosticada pelo exame clínico. Da mesma forma, a história e o exame físico são suficientes para diferenciar hemangioma das malformações vasculares em cerca $95 \%$ dos $\operatorname{casos}^{1}$. Outros tumores de natureza vascular como o Sarcoma de Kaposi podem ser incluídos no diagnóstico diferencial, sobretudo nos pacientes de risco.

Os hemangiomas podem apresentar complicações como ulceração, hemorragia, infecção, trombocitopenia, promover estados de alto débito cardiovascular, deformidades estéticas e comprometimento funcional da via aérea, digestiva, aparelho fonatório, etc. A localização, o tamanho da lesão, as características histológicas, o comportamento citodinâmico são alguns dos fatores que podem influenciar 0 aparecimento daquelas morbidades.

\section{DISCUSSÃO}

À luz da literatura revisada, conclui-se que o caso em estudo é atípico. 0 hemangioma raramente é observado comprometendo exclusivamente a úvula. Algumas características demográficas do paciente, que incluem raça, faixa etária e sexo, também não são as mais freqüentemente observadas no hemangioma.

0 crescimento angiomatoso patológico na úvula, com início na idade adulta, num período relativamente curto, poderia resultar de uma malformação vascular prévia, venosa, com expansão rápida a partir de traumatismo local, infecção ou alterações hormonais conforme descrito anteriormente, ou um hemangioma com repercussão clínica num adulto jovem. No presente caso, o exame clínico sugeriu fortemente tratar-se de hemangioma cavernoso de úvula.

Embora o sarcoma de Kaposi faça parte do diagnóstico diferencial das lesões vasculares da cavidade oral ou orofaringe num adulto de risco, os antecedentes pessoais e o exame físico não sugeriam este diagnóstico, sendo esta impressão corroborada com a sorologia negativa para o HIV.

Portanto, o paciente em estudo apresenta uma lesão de natureza vascular numa localização incomum, com desenvolvimento progressivo, e complicada com o comprometimento funcional da deglutição associado à sensação de corpo estranho na garganta.

A semiologia complementar na avaliação de um hemangioma pode incluir um estudo de imagem por tomografia computadorizada, ressonância magnética (RM), angioressonância ou arteriografia. A ultra-sonografia com 
estudo doppler do fluxo intravascular pode estar indicado em alguns casos e fornecer informações úteis sobre limites da lesão e fluxo venoso intra-tumoral, quando executado por mãos experientes. A tomografia computadorizada pode ser particularmente útil na avaliação de lesões intra-ósseas. No caso em estudo, o exame clínico não sugeriu necessidade de investigação complementar mais extensa, limitada apenas à tomografia computadorizada. A arteriografia convencional expõe detalhadamente o leito vascular e pode ser usada terapeuticamente para embolização seletiva. Porém, a imagem por RM apresenta elevada acurácia na avaliação dessas lesões vasculares, delimitando-as e sugerindo informações a respeito do fluxo vascular.

Por sua vez, o tratamento dos hemangiomas é variável e deve ser particularizado. As opções terapêuticas habitualmente descritas incluem a simples observação, cirurgia, embolização definitiva ou pré-operatória, laser, crio, radio ou escleroterapia, além de farmacoterapia com corticoesteróides ou agentes fibrinolítico $\mathrm{S}^{3,4,5}$.

No presente caso, a uvulectomia total com bisturi de radiofreqüência foi realizada com segurança, então facilitada pela relativa circunscrição do tumor, com mínimo sangramento, resultando na imediata e completa remissão dos sintomas. 0 estudo anatomopatológico confirmou a suspeita diagnóstica de hemangioma cavernoso.

\section{COMENTÁRIOSFINAIS}

Embora de ocorrência rara, o hemangioma de úvula, que tem diagnóstico diferencial com malformação vascular e outros tumores angiogênicos, além de potenciais dificuldades à abordagem cirúrgica, pode determinar sintomas orofaríngeos obstrutivos e representar um risco iminente de sangramento, sobretudo se extenso e não limitado àquele órgão. No comprometimento circunscrito àúvula, a ressecção cirúrgica do hemangioma com radiofreqüência mostrou-se uma segura e efetiva alternativa terapêutica.

\section{AGRADECIMENTOS}

Agradecemos aos doutores Paulo Athanázio e Daniel Athanázio do Serviço de Anatomia Patológica do Hospital das Clínicas da Universidade Federal da Bahia a preciosa colaboração no preparo fotográfico e seleção das lâminas.

\section{REFERÊNCIASBIBUOGRÁFICAS}

1. McGill TJI, Forsen JW, Mulliken JB. Hemangiomas and vascular anomalies of the head and neck. In: Cummings CW, Fredricson JM, Harker LA, Kauser CJ, Richardson MA, Schuller DE. Otolaryngology-Head \& Neck Surgery (Pediatric). $3^{\text {rd }}$ edition. St. Louis: Mosby; 1998. p. 66-80.

2. Carmi G. Clinical and histopatological considerations on an unusual case of hemangioendotelioma of uvula. Otorinolaringol Ital 1967;36(4):319-32.

3. Murphy JB. The management of a large hemangioma of oral cavity with cryoterapy. J Oral Med 1976;33(3):104-6.

4. Waner M, Suen JY, Dinehart S. Treatment of hemangiomas of head and neck. Laryngoscope 1992;102:1123-32.

5. Bartlett JA, Riding KH, Salkeld LJ. Management of hemangiomas of the head and neck in children. J Otolaryngol 1988;17(2):11120

6. Mulliken JB, Glowacki J. Hemangiomas and vascular malformations in infants and children: a classification based endothelial characteristics. Plast Reconstr Surg 1982;69: 412-18.

7. Hassard AD, Byrne BD. Arteriovenous malformations and vascular anatomy of the upper lip and soft palate. Laryngoscope 1985;95:829-32. 